\title{
BMJ Open Half-dose fulvestrant plus anastrozole as a first-line treatment for hormone receptor-positive metastatic breast cancer: a cost-effectiveness analysis
}

\author{
Xiaoting Huang, ${ }^{1}$ Xiuhua Weng, ${ }^{1}$ Shen Lin, ${ }^{1}$ Yiwei Liu, ${ }^{1}$ Shaohong Luo, ${ }^{1}$ \\ Hang Wang, ${ }^{1}$ Wai-kit Ming, ${ }^{2,3}$ Pinfang Huang (i) ${ }^{1}$
}

To cite: Huang $X$, Weng $X$, Lin S, et al. Half-dose fulvestrant plus anastrozole as a first-line treatment for hormone receptorpositive metastatic breast cancer: a cost-effectiveness analysis. BMJ Open 2020;10:e036107. doi:10.1136/ bmjopen-2019-036107

- Prepublication history and additional material for this paper are available online. To view these files, please visit the journal online (http://dx.doi. org/10.1136/bmjopen-2019036107).

$\mathrm{XH}$ and $\mathrm{XW}$ are joint first authors.

Received 30 November 2019 Revised 05 March 2020 Accepted 29 May 2020

\section{Check for updates}

\section{(c) Author(s) (or their} employer(s)) 2020. Re-use permitted under CC BY-NC. No commercial re-use. See rights and permissions. Published by BMJ.

1Department of Pharmacy, The First Affiliated Hospital of Fujian Medical University, Fuzhou,

Fujian, China

${ }^{2}$ Department of Public Health and Preventive Medicine, School of Medicine, Jinan University, Guangzhou, Guangdong, China ${ }^{3}$ Harvard Medical School, Harvard University, Boston, MA, United States

Correspondence to Professor Pinfang Huang; abstract2016@163.com and Professor Wai-kit Ming; wkming@connect.hku.hk

\section{ABSTRACT}

Objective The S0226 trial demonstrated that the combination of half-dose fulvestrant (FUL) and anastrozole (ANA) (F\&A) caused a significant improvement in overall survival (OS) versus ANA monotherapy for first-line treatment of postmenopausal women with hormone receptor-positive metastatic breast cancer (PMW-MBC $(\mathrm{HR}+))$. The objective of this study was to evaluate the cost-effectiveness of F\&A in the first-line treatment for PMW-MBC $(\mathrm{HR}+)$ in China.

Design We constructed a Markov model over a lifetime horizon. The clinical outcomes and utility data were obtained from published literature. Cost data were obtained from official Chinese websites. Sensitivity analyses were performed to test result uncertainty. Setting Chinese healthcare system perspective. Population A hypothetical cohort of adult patients presenting with PMW-MBC ( $\mathrm{HR}+)$.

Interventions F\&A compared with full-dose FUL and ANAmonotherapy.

Main outcome measures The main outcome of this study was the incremental cost-effectiveness ratio (ICER) and quality-adjusted life-years (QALY).

Results ANA was estimated to have the lowest cost and minimum life-years. The ICER of F\&A versus ANA was US\$15 665.891/QALY with incremental cost and QALY of US\$12 401.120 and 0.792, respectively, which was less than the willingness-to-pay of US\$29 383/QALY. Compared with F\&A, FUL yielded a higher cost and a shorter lifetime; hence, it was identified as a dominated strategy. The univariate sensitivity analysis indicated the price of FUL was the most influential factor in our study. The probability that F\&A was cost-effective at a threshold of US\$29 383/ QALY in China was $86.5 \%$.

Conclusion F\&A is a cost-effective alternative to FUL and ANA monotherapy for the first-line treatment of PMW-MBC $(\mathrm{HR}+)$ in China. F\&A is a promising first-line treatment for PMW-MBC $(\mathrm{HR}+)$, and more research is needed to evaluate the economy of using $F \& A$ in other countries.

\section{INTRODUCTION}

The Global Burden of Disease 2015 study reported that breast cancer $(\mathrm{BC})$ is the most commonly diagnosed cancer and is the leading cause of cancer death among
Strengths and limitations of this study

- This paper presents a cost-effectiveness analysis (CEA) comparing half-dose fulvestrant (FUL) plus anastrozole (ANA) (F\&A) with full-dose FUL and ANA monotherapy as the first-line treatment for postmenopausal women with hormone receptor-positive metastatic breast cancer.

- This is the first CEA of F\&A in the Chinese context.

- We have included all relevant outcomes, and we have calculated the outcome data rigorously.

- We used nationally representative data sources to increase generalisability, and performed sensitivity analyses to quantify the uncertainty in our costeffectiveness estimates.

- The Chinese healthcare system perspective may limit the applicability of the findings to other country settings.

women worldwide. ${ }^{1-3}$ There were 2.1 million new cases of $\mathrm{BC}$ in women around the world, with 620000 deaths in $2018 .{ }^{1-3}$ In China, the number of new cases of BC reached 278900 , accounting for $13.28 \%$ of the global total. ${ }^{4}$ Approximately $60 \%$ of new BC cases in postmenopausal women (PMW) are hormone receptor-positive $(\mathrm{HR}+)$ metastatic $\mathrm{BC}$ $(\mathrm{MBC}) .{ }^{5}$ In the recent years, the incidence and mortality of $\mathrm{BC}$ have witnessed a dramatic increase. Consequently, the management of PMW with $\mathrm{HR}+\mathrm{MBC}$ accounts for a large proportion of healthcare budgets. In China, the management of PMW-MBC (HR+) accounts for approximately $3 \%$ of all cancer expenditure. $^{6}$ The economic burden of PMW-MBC (HR+) in women has been gradually increasing globally and has become an issue of prime concern. ${ }^{7}$

For PMW-MBC $(\mathrm{HR}+)$, endocrine therapy plays a crucial role in the initial management. According to current guidelines, ${ }^{8-11}$ first-line treatments include aromatase inhibitors (AI), 
such as anastrozole (ANA), letrozole and exemestane, and fulvestrant (FUL), which are recommended as monotherapies. ANA is the most commonly used AI with a median overall patient survival (mOS) of 41.3 months, ${ }^{12}$ while the mOS for FUL monotherapy approached 54 months. ${ }^{13}$ However, clinical resistance to AI due to oestrogen receptor (ER) hypersensitivity eventually occurs in most patients, leading to disease progression. ${ }^{11}$

Recent studies have shown that the combination of half-dose FUL and ANA (F\&A) is a tolerable, safe, and efficacious alternative to ANA or FUL monotherapy for HR+ MBC. ${ }^{1415}$ The results of a randomised phase III study (S0226 trial) ${ }^{16}$ published in the New England Journal of Medicine on 28 March 2019 demonstrated that in endocrine therapy-naive PMW-MBC $(\mathrm{HR}+)$, the combination of F\&A prolonged OS by 11.9 months compared with ANA monotherapy (HR 0.73; $95 \%$ CI 0.58 to 0.92 ).

In addition to providing health benefits, F\&A versus ANA or FUL monotherapy would change drug expenditures. The extent to which these additional costs are considered a judicious use of resources was evaluated by comparing the costs and effectiveness of competing treatment strategies and summarising the incremental cost per unit of health gained. The economic value of the combination therapy in this patient population has not been evaluated in the published literature.

In order to identify the cost-optimal regimen that produces a better outcome at a lower price, we developed a Markov model to evaluate the cost-effectiveness of F\&A versus FUL and ANA monotherapy from Chinese healthcare system perspectives. We expect that the results of our cost-effectiveness assessment will provide guidance to physicians and healthcare policy-makers in choosing the optimal treatment regimen for PMW-MBC (HR+).

\section{MATERIALS AND METHODS}

\subsection{Model overview}

A Markov model was developed to compare the costs and effectiveness of $\mathrm{F} \& \mathrm{~A}^{16}$ combination therapy with $\mathrm{FUL}^{8}$ and $\mathrm{ANA}^{9}$ monotherapy in PMW-MBC (HR+) patients. The inclusion population was consistent with the S0226 and FIRST trials: (1) PMW-MBC (HR+); (2) no previous chemotherapy; (3) no hormonal therapy and (4) no immunotherapy for metastatic disease was allowed. The patients received one of the following therapeutic regimens: (1) F\&A (ANA $1 \mathrm{mg}$ daily plus FUL $500 \mathrm{mg}$ day $1,250 \mathrm{mg}$ day 14 and 28 , and $250 \mathrm{mg}$ every 28 days after that); (2) FUL (500 mg day 1, 14 and 28, and every 28 days after); (3) or ANA (1 mg daily). ANA is the most commonly used and cost-effective compared with letrozole and exemestane in China, so we selected ANA as control group.

The analysis was conducted from Chinese healthcare system perspective with a lifetime horizon. Only direct costs were considered. The length of each Markov cycle was 28 days, and a half-cycle correction was applied to the model. The costs and effectiveness outcomes were

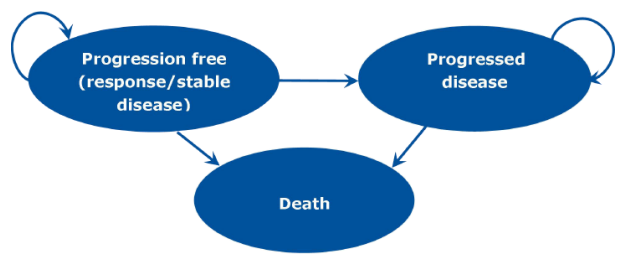

Figure 1 Markov state transition model. Progression free is split into two substates and patient transition between complete response/partial response and stable disease. A three-health-state Markov model was developed as follows: progression free, progression of disease (PD) and death. At the beginning of each Markov cycle, all patients entered the model in the progression free with stable disease state and immediately commenced treatment. From this state, patients could achieve a complete or partial response to therapy and enter progression free with response, experience progression and enter PD, or die and enter death. Patients in the PD could either remain in progressive state or transition to death.

discounted at 3\% annually. A three-health state Markov model was developed as follows: (1) responsive/stable disease; (2) progression of disease (PD) and (3) death (figure 1).

\subsection{Patient and public involvement}

No patient involved.

\subsection{Model assumptions}

The Markov model incorporates several key assumptions:

- At the beginning of each Markov cycle, patients in the responsive/stable state could remain in one of three states: (1) remain in a responsive/stable state; (2) transition to the progressive state or (3) transition to death. Patients in the progressive state could either remain in progressive state or transition to death.

- Patients received the initial treatment (such as F\&A, ANA or FUL) when they were in the responsive/ stable disease state and discontinued the treatment when they transitioned to the progressive state. All patients have good compliance in any state.

- According to the current guideline, ${ }^{8}$ after disease progression, patients without visceral crisis received second-line hormonal therapy (including AI plus cyclin-dependent kinases 4 and 6 (CDK4/6) inhibitor, FUL plus CDK4/ 6 inhibitor and everolimus), for those with visceral crisis received chemotherapy. The progressive medication costs was based on an average cost of all kinds of strategies (table 1).

- In the model, probabilities from progression free state to death were assumed to be the same as the natural mortality rate, obtained as the age-specific and sexspecific death rate from the life tables for the Chinese populations. ${ }^{17}$

\subsection{Model Inputs}

\subsection{1 clinical effectiveness}

Kaplan-Meier survival curves for progression-free survival (PFS) and OS were derived from a phase III trial of F\&A versus ANA $(\mathrm{S} 0226, \mathrm{NCT} 00075764)^{16}$ and a phase II 
Table 1 Postprogression medication costs

\begin{tabular}{|c|c|c|c|}
\hline Medication & Dose & Monthly cost (US\$) & References \\
\hline \multicolumn{4}{|l|}{ Hormonal therapy } \\
\hline Anastrozole+abemaciclib & $1 \mathrm{mg}$ qd+200 mg two times per day & 12421.49 & 819 \\
\hline Anastrozole+ribociclib & $1 \mathrm{mg} \mathrm{qd+600mg} \mathrm{q1-21d}$ & 15890.9 & 819 \\
\hline Letrozole+abemaciclib & $2.5 \mathrm{mg} q d+200 \mathrm{mg}$ two times per day & 12437.11 & 819 \\
\hline Letrozole+ribociclib & $2.5 \mathrm{mg} q \mathrm{~d}+600 \mathrm{mg}$ q1-21d & 15906.52 & 819 \\
\hline Exemestane+abemaciclib & $25 \mathrm{mg}$ qd+200 mg two times per day & 12434.39 & 819 \\
\hline Exemestane+palbociclib & $25 m g$ qd+125mg q1-21d & 12538.55 & 819 \\
\hline Exemestane+ribociclib & $25 \mathrm{mg} \mathrm{qd}+600 \mathrm{mg} \mathrm{q1-21d}$ & 15903.8 & 819 \\
\hline Fulvestrant+abemaciclib & $\begin{array}{l}500 \mathrm{mg} \text { q28d+200 mg two times per } \\
\text { day }\end{array}$ & 13659.2 & 819 \\
\hline Everolimus & $10 \mathrm{mg}$ qd & 1199.9 & 819 \\
\hline Average & & 12950.06 & \\
\hline \multicolumn{4}{|l|}{ Chemotherapy } \\
\hline Fluorouracil & $600 \mathrm{mg} / \mathrm{m}^{2}$ every 3 weeks & 41.97 & 819 \\
\hline Epirubicin & $60 \mathrm{mg} / \mathrm{m}^{2}$ every 3 weeks & 167.51 & 819 \\
\hline Cyclophosphamide & $600 \mathrm{mg} / \mathrm{m}^{2}$ every 3 weeks & 9.82 & 819 \\
\hline Capacitabine & $1250 \mathrm{mg} / \mathrm{m}^{2} /$ day & 235.33 & 819 \\
\hline Paclitaxel & $80 \mathrm{mg} / \mathrm{m}^{2}$ & 1435.56 & 819 \\
\hline
\end{tabular}

trial of FUL versus ANA (FIRST, NCT01602380). ${ }^{13}$ The Weibull distribution ${ }^{18}$ was fit to a Kaplan-Meier survival curve, and the R-Studio software was used to estimate scale $(\lambda)$ and shape $(\gamma)$ parameters. These parameters were substituted into the formula: $\mathrm{P}(\mathrm{t})=1-\exp \left(\lambda[\mathrm{t}-1]^{\gamma_{-}}\right.$ $\left.\lambda \mathrm{t}^{\gamma}\right)$ to calculate the transition probability. The scale and shape in Weibull regression with their corresponding ranges used in sensitivity analyses were in the table 2 . The transition probability of each treatment scheme in each state of each cycle in online supplementary table 1.

\subsection{2costs and utilities}

Literature search on PubMed, Cochrane Library and EMBASE over the period 2000 to present was performed

Table 2 Key clinical and health parameters

\begin{tabular}{lll} 
& PFS (credible intervals) & OS (credible intervals) \\
\hline Weibull scale for F\&A & $0.0758(0.0739-0.0777)$ & $0.0009(0.0006-0.0012)$ \\
Weibull shape for F\&A & $0.7776(0.7703-0.7894)$ & $1.1094(1.0936-1.1252)$ \\
$R^{2}$ for F\&A & 0.9959 & 0.9922 \\
Weibull scale for FUL & $0.0272(0.0265-0.0279)$ & $0.0045(0.0043-0.0046)$ \\
Weibull shape for FUL & $1.1029(1.0950-1.1107)$ & $1.3391(1.3292-1.3491)$ \\
$R^{2}$ for FUL & 0.9946 & 0.9901 \\
Weibull scale for ANA & $0.0598(0.0582-0.0615)$ & $0.0073(0.0072-0.0075)$ \\
Weibull shape for ANA & $0.9581(0.9485-0.9677)$ & $1.2327(1.2267-1.2387)$ \\
$R^{2}$ for ANA & 0.9922 & 0.9972 \\
\hline
\end{tabular}

ANA, anastrozole; F\&A, half-dose fulvestrant plus anastrozole treatment group; FUL, fulvestrant; OS, overall survival; PFS, progression-free survival. 
using keywords 'FUL', 'AI', 'MBC', 'cost-effectiveness', 'economic evaluation' and 'China'. A manuscript was included if it had data relevant to the model inputs. If a model parameter has multiple sources, the weighted average was used as the base case value, and the high/low values formed the sensitivity analysis range.

The costs were estimated from Chinese healthcare system perspective. Only direct medical costs were considered in the model, including the drug, management of treatment-related serious adverse events (SAEs) and routine disease management. The price of endocrine drugs and chemotherapy drugs was obtained from the median price of the national drug winning bid in 2019. We searched the price in Yaozh (https://yaozh.com/), which is a Big Data service platform for the Chinese healthcare industry and provides information on the pharmaceutical industry, including the bidding information of medicines in all the Chinese provinces of China. ${ }^{19}$ Routine disease management costs, including hospitalisations, outpatient visits and laboratory tests, were modelled using data from published literature and applied monthly to the progression-free and PD states. ${ }^{19-21}$ And the other medical costs were derived from previously published studies. ${ }^{21}$ Costs from past sources were adjusted to 2019 US dollars according to Chinese Consumer Price Index healthcare services group..$^{22}$ The Chinese yuan (CNY) was converted to the US dollar based on an exchange rate in 2019 of CNY6.89=US $\$ 1.00 .^{23}$

The utility values were calculated according to published utilities derived by using Visual Analogue Scale and standard gamble. ${ }^{24-27}$ In addition to utility associated with each health state, disutilities (or quality of life (QOL) impairment) associated with SAEs were also considered in the base case. Disutility values for each SAE were also obtained from the literature. All information regarding the costs, utility and the disutility values associated with SAEs are listed in table 3.

\subsection{Cost-effectiveness analysis}

Model outputs included total costs and total effectiveness measured according to quality-adjusted life-years (QALYs) associated with each treatment arm. QALYs were estimated as the time spent in each state weighted by the utility of each state. Incremental cost-effectiveness ratios (ICERs) measured as incremental costs per QALY gained were estimated, by comparing F\&A with each alternative endocrine monotherapy. Treatment that is more effective and more costly was considered cost-effective if the ICER was less than a willingness-to-pay (WTP) threshold of US\$29 383/QALY, which is set as three time the per capita gross domestic product of China in 2018 according to WHO recommendation for cost-effectiveness analysis (CEA). ${ }^{22}$ The Markov model was implemented using Tree Age Pro 2017 (Tree Age Software).

\subsection{Sensitivity Analysis}

A series of sensitivity analyses were performed to test the robustness of the model. In the univariable sensitivity analysis, all variables differed over a plausible range, obtained from credible intervals or by assuming a variance of $20 \%$ from base-case values. ${ }^{28}{ }^{29}$ We performed 10000 Monte Carlo simulations for conducting probabilistic sensitivity analyses with the variables concurrently varied with a specific pattern of distribution as shown in table 3 .

\section{RESULTS}

\subsection{Base-case analysis}

The result of the cost and health outcome estimated using the model with a life-time horizon is presented in table 4. The result showed that ANA had the lowest cost (US\$45 520.641) with minimum life-years (LYs) of 4.562. The model projected that the life expectancy of patients receiving F\&A was $5.728 \mathrm{LYs}$, which was 0.35 and 1.166 LYs more than that of patients receiving FUL and ANA, respectively. Accounting for QALYs, the incremental number of QALYs increased with F\&A compared with FUL and ANA in the base case was 0.144 and 0.792 , respectively.

In contrast to ANA, F\&A cost an additional US $\$ 12$ 401.12, yielding an ICER of US\$15 665.89/QALY, which was less than the WTP of US $\$ 29383$ per QALY gained in China. Compared with FUL, F\&A was superior to FUL with a cost-savings of US\$12260.783 and gain of 0.144 QALYs; hence, it was identified as a dominated strategy. This main finding indicated that the F\&A therapeutic strategy was a cost-effective approach in China.

In the cohort analysis, 10000 samples were simulated in the FUL, ANA and F\&A, respectively. In the 30th cycle (about 2.3 years), 3052, 2058 and 3343 samples were in progressive-free state, respectively. (online supplementary table 1)

\subsection{Sensitivity analyses}

\subsubsection{Univariable sensitivity analysis}

Figure 2 presents the results of the univariable sensitivity analysis for the model in China, respectively. The cost of FUL had the greatest impact on ICER in China. Other variables, including the $\mathrm{PD}$ health state costs and utility for PFS had a moderate or minor influence on the ICER. All variables resulted in an ICER value lower than the WTP in China. The results of the univariable sensitivity analysis confirmed that our model was robust.

\subsection{2probabilistic sensitivity analysis}

A cost-effectiveness acceptability curve was constructed to present the results of the probabilistic sensitivity analysis (figure 3). In China, compared with FUL and ANA, the probability that F\&A was cost-effective at a WTP for a QALY of US $\$ 29383$ was estimated to be $86.5 \%$. Therefore, $\mathrm{F} \& \mathrm{~A}$ was the most likely treatment to be cost-effective in China.

\section{DISCUSSION}

Both FUL and ANA are well-documented drugs in terms of efficacy, tolerability and safety in PMW-MBC 
Table 3 Sensitivity analysis parameter's ranges and distribution

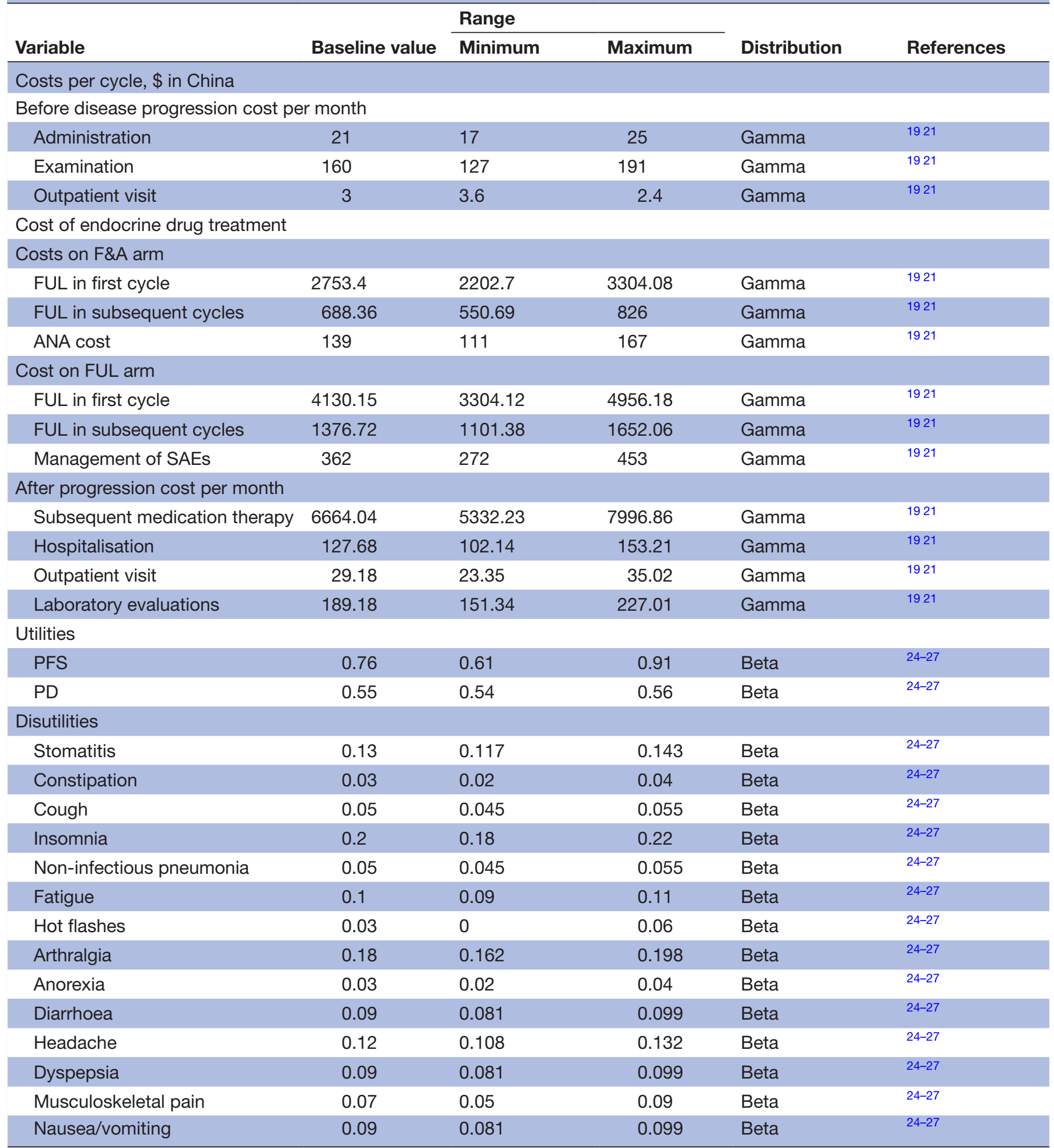

ANA, anastrozole; ANA, anastrozole; F\&A, half-dose fulvestrant plus anastrozole treatment group; F\&A, half-dose fulvestrant plus anastrozole treatment group; FUL, fulvestrant;FUL, fulvestrant;PD, progressed disease; PD, progressed disease; PFS, progression free survival; PFS, progression-free survival; SAE, serious adverse event; SAE, serious adverse event.

$(\mathrm{HR}+) .{ }^{12} 141726$ FUL and ANA are included in the international treatment guidelines as the recommended endocrine monotherapies in MBC for first-line and later-line treatments. ${ }^{8-10}$ However, long-term use of ANA monotherapy leads to clinical resistance in most patients, which eventually leads to disease progression. Some clinical studies have found that adding the selective ER downregulator FUL to ANA therapy is more effective than treatment with ANA alone, considering that one of the resistance mechanisms to ANA is chronic stimulation of 
Table 4 Summary of cost and outcome results in China

\section{Treatment}

\section{Incremental}

\begin{tabular}{|c|c|c|c|c|c|}
\hline Results & ANA & $F \& A$ & FUL & F\&A versus ANA & F\&A versus FUL \\
\hline Total cost, US\$ & 45520.64 & 57921.76 & 70182.54 & 12401.12 & -12260.78 \\
\hline Overall LYs & 4.562 & 5.728 & 5.378 & 1.166 & 0.35 \\
\hline \multicolumn{6}{|l|}{ ICER, US\$ } \\
\hline Per LY & & & & 10635.61 & -350308.09 \\
\hline
\end{tabular}

F\&A, half-dose fulvestrant plus anastrozole treatment group; FUL, fulvestrant;

ANA, anastrozole; LYs, life-years; QALYs, quality-adjusted life years;

ICER, incremental cost-effectiveness ratio;

\section{(A) Tomado Analysis (ICER of F\&A vs. FUL)}

FUL in subsequent cycles cost on FUL, $\$$ FUL in subsequent cycles cost on F\&A,\$ PD cost,\$ FUL in first cycle cost on FUL, \$ Discount factor, \% FUL in first cycle cost on F\&A,\$ Utility for PFS Utility for PD Tests cost per cycle,\$ Utility for Arthralgia Administration per cycle cost,\$ Utility for Hot flashes Utility for Fatigue Utility for Nausea

$\begin{array}{lllll}-160000-140000-120000-100000-80000 & -60000 & -40000 & -20000\end{array}$

ICER,\$/QALY

(B) Tomado Analysis (ICER of F\&A vs. ANA)

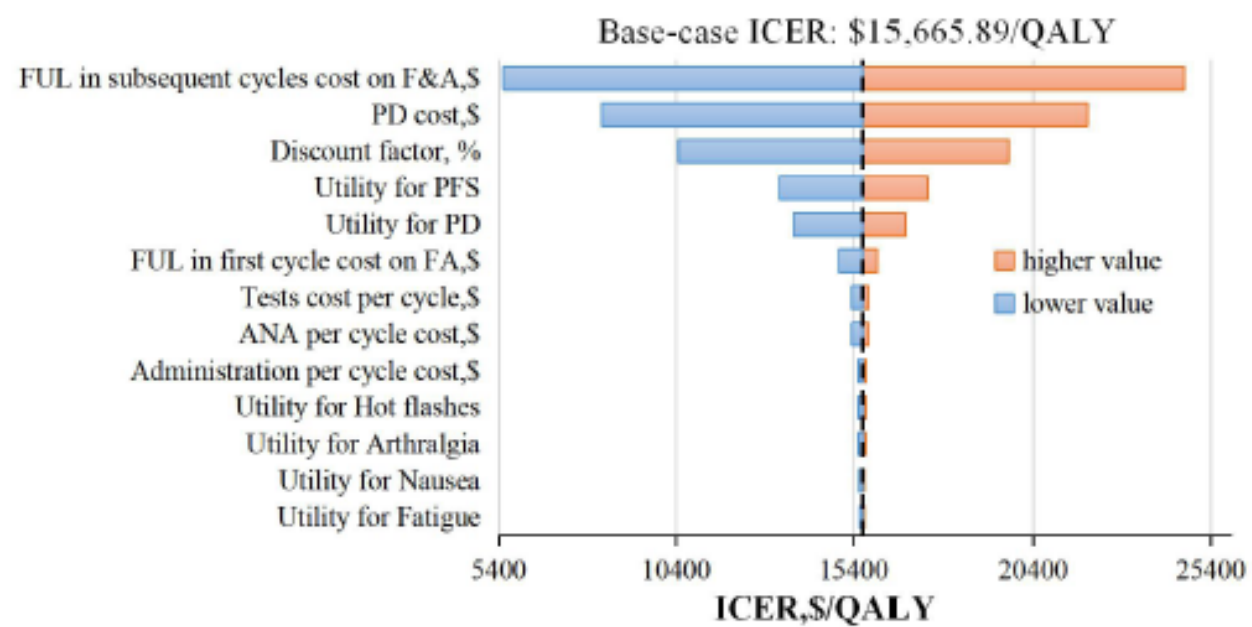

Figure 2 The results of univariable sensitivity analysis in China: (A) F\&A versus FUL; (B) F\&A versus ANA. This diagram shows incremental cost-effectiveness ratio (ICER) of F\&A versus FUL $500 \mathrm{mg}$ versus ANA for different model input parameters. For example, F\&A compared with ANA, the dotted line intersecting the sky blue and orange bars represents the ICER of US\$15665.89 per quality-adjusted life-year (QALY) in China from the base case results. ANA, anastrozole; F\&A, fulvestrant plus anastrozole treatment group; FUL, fulvestrant; PFS, progression-free survival; PD, progressed disease. 


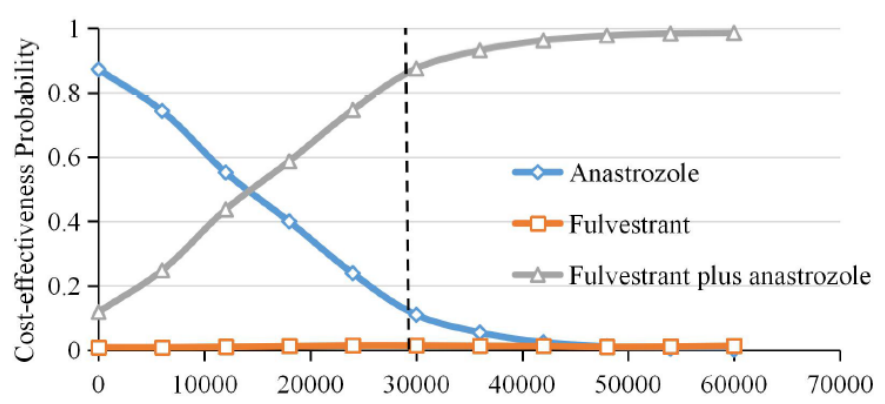

Willingness to Pay \$ per QALY

Figure 3 The cost-effectiveness acceptability curve in China. Results of the probabilistic sensitivity analyses based on 10000 Monte-Carlo simulations, which involves sampling model variable values from distributions imposed on variables to indicate uncertainty about whether F\&A are cost-effective at different willingness-to-pay thresholds. The probabilities that F\&A was cost-effective at thresholds of US\$29383 per QALY in China was $86.5 \%$. QALY indicates quality-adjusted life-year. F\&A, fulvestrant plus anastrozole treatment group; QALY, quality-adjusted life-year.

ERs by low levels of estradiol. Recently, the latest results of the randomised phase III study (S0226 trial) published in the New England Journal of Medicine on 28 March 2019 clearly demonstrated that F\&A had a greater clinical efficacy than ANA monotherapy did for the first-line treatment of $\mathrm{HR}+\mathrm{MBC}$.

Previous studies have assessed the cost-effectiveness of FUL versus ANA monotherapy in first-line treatment of MBC in China, ${ }^{17} 30$ whereas no economic evaluation of the combination of aforementioned endocrine therapy in PMW-MBC $(\mathrm{HR}+)$ has been published in the literature. In the absence of direct head-to-head studies of FUL versus F\&A, we conducted indirect comparisons of the S0226 and FIRST trial results with the same enrolled and exclusive criteria. The FIRST trial showed that FUL has superior efficacy and is a preferred treatment option for patients with $\mathrm{HR}+\mathrm{MBC}$ compared with ANA. However, the final OS results of the FIRST study and S0226 trial suggested that FUL monotherapy yielded a slightly longer OS than F\&A did (54 and 52 months, respectively). The different observation duration in these two trials made it difficult to compare the efficacy of FUL and F\&A. ${ }^{21}$ Thus, we conducted a Markov model to measure whether F\&A could outperform FUL monotherapy in efficacy and subsequent cost-effectiveness. Meanwhile, we performed a CEA between F\&A and ANA.

$\mathrm{BC}$ constitutes one of the most expensive malignancies to treat, however, the proportion of Chinese healthcare in fiscal expenditure is relatively low worldwide. The economic burden of BC has been gradually increasing and has become an issue of prime concern. Unlike the other countries, ${ }^{31}$ the pricing mechanisms in China have gradually changed to multilateral negotiations since 2015 and economic evaluation is an important content during the multilateral negotiations. ${ }^{32}$ Among the current anti-BC drugs, the price of FUL is relatively high. Moreover, in
2019, FUL withdrew from the list of Chinese medical insurance due to the failure of medical insurance negotiation, which result in lower availability for many patients. Considering special medical environments, the high cost of FUL, the limits of national medical resources and the heavy financial burden of anticancer treatment in China, it is imperative to evaluate the economics of additional using half-dose FUL. We conducted this study immediately after the results of the S0226 trial ${ }^{16}$ were published. Our results of cost-effectiveness of FUL are helpful for policy-maker to consider relist FUL to Chinese medical insurance catalogue, which is adjusted every 2 years in order to expend more economical. Meanwhile, our univariable sensitivity suggested the price of FUL is an important indicator, and lower price would be a potential measure to improve FUL cost-effectiveness furtherly, which is helpful to inform the multilateral drug price negotiations that may be upcoming for FUL.

Most clinical trials have limited observation. Using Markov model combined with clinical trial data, estimating the long-term effects of clinical intervention can provide valuable information for clinical decision-makers. We used Markov model to extrapolate the efficacy of FUL, ANA and F\&A regimens for patients longer than the time of clinical trials. The model results suggested that F\&A could be more cost-effective than FUL or ANA monotherapy is in China. F\&A was superior to FUL with costsavings and acquisition of QALYs, and compared with ANA, the F\&A ICER were US\$15 665.89/QALY. In addition, both univariate sensitivity and probabilistic sensitivity analyses demonstrated that the model results were robust for the uncertainty of model input parameters. All variables resulted in an ICER value lower than a WTP threshold of US\$29383 per QALY in China. The main factor influencing our model was the cost of FUL. For the base-case analysis, we applied the brand of FUL with the highest price (patent drug) in China. After patent expires, the cost of FUL will definitely reduce, which will further improve the cost-effectiveness of two regimens which are F\&A and FUL.

As with any model, there are some limitations to our analysis. First, the model inputs were obtained from different randomised clinical trials (RCTs) with the same inclusion and exclusion criteria. And the most registered participants were Caucasians and the minority were Asian. But the efficacy of F\&A has shown a slight but insignificant relationship to race, sex and age. ${ }^{14}$ Further, we selected the best fit model input and conducted extensive sensitivity analysis to test the effect of uncertainties on model results. Although variations in ICERs were observed, none of them changed the conclusion of this study. Second, owing to the lack of QOL data, we assumed that healthcare utilities depended on health state and the response rate of each treatment. Nonetheless, we carried out a sensitivity analysis on the utilities, and the tornado analysis showed that utilities were not the main factor in our results, and actually had little effect on the results. Third, the Markov model incorporates several assumptions. The model 
assumes a specific treatment in disease progression, which may differ from clinical practice. However, the model was developed based on the American National Comprehensive Cancer Network (NCCN) guidelines and most oncologists in China make clinical decisions according to NCCN guidelines. ${ }^{33}$ We used the average cost of all kinds of strategies in progressive state as the progressive medication costs. In real-word situation, patients may receive only one second-line treatment, which may have impact on our outcome. While the univariable sensitivity analysis suggested that PD health state costs had slight effect on ICER. In addition, we assumed that all patients have good compliance, which may not be consistent with the actual situation, non-compliance with endocrine therapy drug can have a negative impact on HR+ BC outcome. During its long-term medication, the compliance may lower gradually due to patient's attitudes and beliefs, lack of finances, severe toxic effects and etc, ${ }^{34}{ }^{35}$ which would happen more often in oral medicine ANA than injection FUL. ${ }^{36}$ So the efficacy and cost of ANA and F\&A may be overestimated due to the lower of compliance. Further studies need to incorporate compliance to reflect the outcome of real-world studies. These assumptions may cause bias in model, however, the cohort analysis showed that the model basically simulated the survival characteristics of patients in the trial, which suggested our assumption resulted in little bias on results. The outcome of the cohort analysis predicted the mOS with F\&A, FUL, and ANA to be 52, 54 and 41 months, respectively, which was similar to the results of the RCTs (online supplementary figure 1). The result of the PFS was also similar (online supplementary figure 2), which indicated that the model was reliable. Fourth, we only consider the direct treatment costs. In fact, phenotyping, multilevel diagnostics and multiomic targets in liquid biology play a crucial role before determining the drug treatment plan, and they are also important to improve the cost-effectiveness of MBC treatment and overall management. ${ }^{37}{ }^{38}$ Finally, $\mathrm{BC}$ is a highly heterogeneous disease. In our study, only CEA of patients wih B C with ' $\mathrm{HR}+$ ' status is performed. Using biomarkers based on chronic inflammation to predict and prevent cancer progression, and the use of machine learning multiomic approach are considered to be effective tools for patient stratification. ${ }^{39} 40$ In further research, these methods should be used to classify the risk of patients with $\mathrm{BC}$ to achieve more detailed stratification of patient characteristics for CEA.

\section{CONCLUSIONS}

Despite its limitations, the results of the present CEA suggest that half-dose FUL plus ANA appears to be a costeffective alternative to FUL and ANA monotherapy for the first-line treatment of PMW-MBC ( $\mathrm{HR}+)$ in China, which could provide appropriate information for decision makers in the healthcare system and the upcoming multilateral drug price negotiations. With the sharp rise of drug prices, it is particularly important that insurance decision-making and pricing should be guided by CEA, especially in China, where the insurance coverage is insufficient. F\&A is a promising first-line treatment for PMW$\mathrm{MBC}(\mathrm{HR}+)$, and more research is needed to evaluate the economy of using F\&A in other countries. Meanwhile, the application of related technologies in the diagnosis and treatment of $\mathrm{BC}$, such as phenotyping, multilevel diagnostics and multiomics targets of liquid biopsy also play a crucial role in improving the cost-effectiveness of MBC treatment and overall management, which should be a new topic worth studying.

Contributors $\mathrm{XH}$ and XW designed the study. All authors contributed to the cost-effectiveness model: XH initiated the model, SL, YL, SL, HW, MW-K and PH contributed their expertise in the model building and parameter determination and $\mathrm{XH}$ modified and finalised the model. XH and XW drafted the manuscript and all authors significantly contributed to and reviewed the final manuscript. All authors agreed to be accountable for all aspects of the work.

Funding The authors have not declared a specific grant for this research from any funding agency in the public, commercial or not-for-profit sectors.

Competing interests None declared.

Patient consent for publication Not required.

Provenance and peer review Not commissioned; externally peer reviewed.

Data availability statement All data relevant to the study are included in the article or uploaded as online supplementary information. No additional data are available.

Open access This is an open access article distributed in accordance with the Creative Commons Attribution Non Commercial (CC BY-NC 4.0) license, which permits others to distribute, remix, adapt, build upon this work non-commercially, and license their derivative works on different terms, provided the original work is properly cited, appropriate credit is given, any changes made indicated, and the use is non-commercial. See: http://creativecommons.org/licenses/by-nc/4.0/.

ORCID iD

Pinfang Huang http://orcid.org/0000-0002-2699-9663

\section{REFERENCES}

1 Global Burden of Disease Cancer Collaboration, Fitzmaurice C, Allen $\mathrm{C}$, et al. Global, regional, and national cancer incidence, mortality, years of life lost, years lived with disability, and disability-adjusted life-years for 32 cancer groups, 1990 to 2015: a systematic analysis for the global burden of disease study. JAMA Oncol 2017;3:524.

2 Bray F, Ferlay J, Soerjomataram I, et al. Global cancer statistics 2018: GLOBOCAN estimates of incidence and mortality worldwide for 36 cancers in 185 countries. CA Cancer J Clin 2018;68:394-424.

3 Kassebaum NJ, Arora M, Barber RM, et al. Global, regional, and national disability-adjusted life-years (DALYs) for 315 diseases and injuries and healthy life expectancy (HALE), 1990-2015: a systematic analysis for the global burden of disease study 2015. The Lancet 2016;388:1603-58.

4 Che Y, You J, Zhou S, et al. Comparison of survival rates between Chinese and Thai patients with breast cancer. Asian Pac J Cancer Prev 2014;15:6029-33.

$5 \mathrm{Li} \mathrm{Cl}$, Daling JR, Malone KE. Incidence of invasive breast cancer by hormone receptor status from 1992 to 1998 . J Clin Oncol 2003;21:28-34.

6 Yin X, Xu Y, Man X, et al. Direct costs of both inpatient and outpatient care for all type cancers: the evidence from Beijing, China. Cancer Med 2019;8:3250-60.

7 Harford J, Azavedo E, Fischietto M, et al. Guideline implementation for breast healthcare in low- and middle-income countries: breast healthcare program resource allocation. Cancer 2008;113:2282-96.

8 National Comprehensive Cancer Network (NCCN). Clinical practice guidelines in oncology-breast cancer, 2019. Available: http://www. nccn.org [Accessed 25 July 2019].

9 Cardoso F, Senkus E, Costa A, et al. 4th ESO-ESMO International Consensus Guidelines for Advanced Breast Cancer (ABC 4). Ann Oncol 2018;29:1634-57. 
10 Senkus E, Kyriakides S, Ohno S, et al. Primary breast cancer: ESMO clinical practice guidelines for diagnosis, treatment and follow-up. Ann Oncol 2015;26 Suppl 5:v8-30.

11 Cardoso F, Costa A, Senkus E, et al. 3rd ESO-ESMO international consensus guidelines for advanced breast cancer (ABC 3). Ann Oncol 2017;28:3110-3111.

12 Mehta RS, Barlow WE, Albain KS, et al. Combination anastrozole and fulvestrant in metastatic breast cancer. $N$ Engl J Med 2012;367:435-44.

13 Ellis MJ, Llombart-Cussac A, Feltl D, et al. Fulvestrant 500 Mg versus anastrozole $1 \mathrm{Mg}$ for the first-line treatment of advanced breast cancer: overall survival analysis from the phase II first study. $J$ Clin Oncol 2015;33:3781-7.

14 Johnston SR, Kilburn LS, Ellis P, et al. Fulvestrant plus anastrozole or placebo versus exemestane alone after progression on non-steroidal aromatase inhibitors in postmenopausal patients with hormonereceptor-positive locally advanced or metastatic breast cancer (SoFEA): a composite, multicentre, phase 3 randomised trial. Lancet Oncol 2013;14:989-98.

15 Graham J, Pitz M, Gordon V, et al. Clinical predictors of benefit from fulvestrant in advanced breast cancer: a meta-analysis of randomized controlled trials. Cancer Treat Rev 2016;45:1-6.

16 Mehta RS, Barlow WE, Albain KS, et al. Overall survival with fulvestrant plus anastrozole in metastatic breast cancer. $N$ Engl J Med 2019;380:1226-34.

17 Ding H, Fang L, Xin W, et al. Cost-effectiveness analysis of fulvestrant versus anastrozole as first-line treatment for hormone receptor-positive advanced breast cancer. Eur J Cancer Care 2017;26:e12733.

18 Weibull CE, Eloranta S, Johansson ALV, et al. Reply to Silvia Selinski, Daniel Ovsiannikov, Jan G. Hengstler and Klaus Golka's letter to the Editor re: Caroline E. Weibull, Sandra Eloranta, Daniel Altman, Anna L.V. Johansson, Mats Lambe. Childbearing and the risk of bladder cancer: a nationwide population-based cohort Study. Eur Urol 2013;64:e81:733-388.

19 Yaozhi Data Service Provider. Drug information inquiry, 2019. Available: https://db.yaozh.com/yaopinzhongbiao [Accessed $1 \mathrm{Apr}$ 2019].

20 Liao X-Z, Shi J-F, Liu J-S, et al. Medical and non-medical expenditure for breast cancer diagnosis and treatment in China: a multicenter cross-sectional study. Asia Pac J Clin Oncol 2018;14:167-78.

21 Sabale U, Ekman M, Thunström D, et al. Economic evaluation of fulvestrant $500 \mathrm{mg}$ compared to generic aromatase inhibitors in patients with advanced breast cancer in Sweden. Pharmacoecon Open 2017;1:279-90.

22 National Bureau of Statistics of China. Annual data. Available: www. stats.gov.cn/english/Statisticaldata/AnnualData/ [Accessed 24 Jun 2019].

23 Exchange rate. Exchange rate inquiry, 2019. Available: http://www. exchangerates24.com/ [Accessed 10 Oct 2019].

24 Lloyd A, Nafees B, Narewska J, et al. Health state utilities for metastatic breast cancer. Br J Cancer 2006;95:683-90.

25 Lloyd A, van Hanswijck de Jonge P, Doyle S, et al. Health state utility scores for cancer-related anemia through societal and patient valuations. Value Health 2008;11:1178-85.
26 McCloskey EV, Hannon RA, Lakner G, et al. Effects of third generation aromatase inhibitors on bone health and other safety parameters: results of an open, randomised, multi-centre study of letrozole, exemestane and anastrozole in healthy postmenopausal women. Eur J Cancer 2007;43:2523-31.

27 Shiroiwa T, Fukuda T, Shimozuma K, et al. Long-term health status as measured by EQ-5D among patients with metastatic breast cancer: comparison of first-line oral S-1 and taxane therapies in the randomized phase III select bc trial. Qual Life Res 2017;26:445-53.

28 Wan X, Zhang Y, Tan C, et al. First-line nivolumab plus ipilimumab vs sunitinib for metastatic renal cell carcinoma: a cost-effectiveness analysis. JAMA Oncol 2019;5:491-6.

29 Wan X, Luo X, Tan C, et al. First-line atezolizumab in addition to bevacizumab plus chemotherapy for metastatic, nonsquamous non-small cell lung cancer: a United States-based cost-effectiveness analysis. Cancer 2019;125:3526-34.

30 Newman MJ, Jones LT, Kraft JM, et al. Cost-effectiveness of fulvestrant $250 \mathrm{Mg}$ versus $500 \mathrm{Mg}$ in postmenopausal women with estrogen receptor-positive metastatic breast cancer and disease progression after antiestrogen therapy. $J$ Oncol Pharm Pract 2012;18:394-401.

31 Saltz LB. Can money really be no object when cancer care is the subject? J Clin Oncol 2015;33:1093-4.

32 Wan XM, Peng LB, Ma JA, et al. Economic evaluation of nivolumab as a second-line treatment for advanced renal cell carcinoma from US and Chinese perspectives. Cancer 2017;123:2634-41.

$33 \mathrm{Gu}$ J, Chen N. Current status of rectal cancer treatment in China. Colorectal Dis 2013;15:1345-50.

34 Hamine S, Gerth-Guyette E, Faulx D, et al. Impact of mHealth chronic disease management on treatment adherence and patient outcomes: a systematic review. J Med Internet Res 2015;17:e52.

35 VanEpps EM, Troxel AB, Villamil E, et al. Financial incentives for chronic disease management: results and limitations of 2 randomized clinical trials with new York Medicaid patients. Am J Health Promot 2018;32:1537-43.

36 Lesur A, Dalenc F, Beguignot M. Consultation dédiée de prescription d'une hormonothérapie adjuvante chez la femme jeune traitée pour cancer du sein hormonosensible : une clé pour améliorer l'adhésion ? Bull Cancer 2019;106:S28-36.

37 Golubnitschaja O, Polivka J, Yeghiazaryan K, et al. Liquid biopsy and multiparametric analysis in management of liver malignancies: new concepts of the patient stratification and prognostic approach. Epma J 2018;9:271-85.

38 Bubnov R, Polivka J, Zubor P, et al. "Pre-metastatic niches" in breast cancer: are they created by or prior to the tumour onset? "Flammer Syndrome" relevance to address the question. Epma J 2017:8:141-57.

39 Fröhlich H, Patjoshi S, Yeghiazaryan K, et al. Premenopausal breast cancer: potential clinical utility of a multi-omics based machine learning approach for patient stratification. Epma J 2018;9:175-86.

40 Qian S, Golubnitschaja O, Zhan X. Chronic inflammation: key player and biomarker-set to predict and prevent cancer development and progression based on individualized patient profiles. Epma $J$ 2019;10:365-81. 